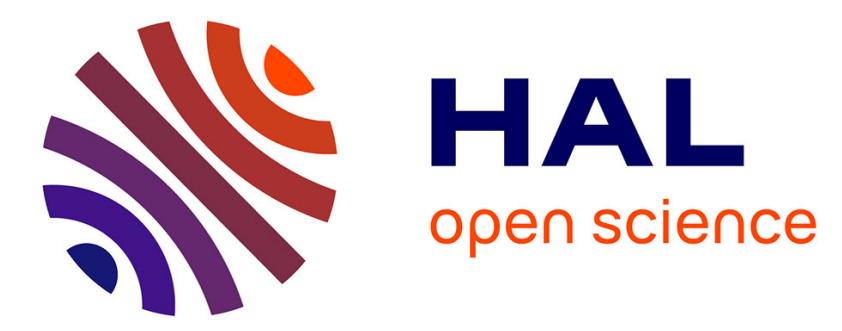

\title{
Electric forces on a confined advacancy island
}

Frédéric Leroy, Ali El-Barraj, Fabien Cheynis, Pierre Müller, Stefano Curiotto

\section{To cite this version:}

Frédéric Leroy, Ali El-Barraj, Fabien Cheynis, Pierre Müller, Stefano Curiotto. Electric forces on a confined advacancy island. Physical Review B, 2020, 102 (23), 10.1103/PhysRevB.102.235412 . hal-02569097v2

\section{HAL Id: hal-02569097 \\ https://hal.science/hal-02569097v2}

Submitted on 10 Dec 2020

HAL is a multi-disciplinary open access archive for the deposit and dissemination of scientific research documents, whether they are published or not. The documents may come from teaching and research institutions in France or abroad, or from public or private research centers.
L'archive ouverte pluridisciplinaire $\mathbf{H A L}$, est destinée au dépôt et à la diffusion de documents scientifiques de niveau recherche, publiés ou non, émanant des établissements d'enseignement et de recherche français ou étrangers, des laboratoires publics ou privés. 


\author{
F. Leroy,${ }^{1, *}$ A. El Barraj,${ }^{1}$ F. Cheynis,${ }^{1}$ P. Muller,${ }^{1}$ and S. Curiotto ${ }^{1}$ \\ ${ }^{1}$ Aix Marseille Univ, CNRS, CINAM, Marseille, France
}

(Dated: November 17, 2020)

\begin{abstract}
The passage of an electric current in a material can cause a biased mass transport at its surface. This migration phenomenon is intimately related to the microscopic details of atomic processes of diffusion and attachment/detachment at step edges. Using low energy electron microscopy we have examined in operando under an electric current the migration of $\mathrm{Si}(111)-1 \times 1$ advacancy islands confined on $\mathrm{Si}(111)-7 \times 7$ terraces. The islands move opposite to the current direction, with velocity increasing with the radius. The effective valence of Si adatoms is $2.8 \pm 0.5$ and the kinetic length of attachment-detachment is about $500 \mathrm{~nm}$. The analysis of the islands shape reveals that the electric current biases significantly the kinetic rate of mass transfers at step edges modifying the overall island shape.
\end{abstract}

$5 \quad$ Advances in the fabrication of nanostructures widely 6 depend on the degree of knowledge of atomic processes 7 at surfaces. In that respect atomic steps, as the most 8 abundant structures at surfaces, play a key role in mass 9 transfers. They are involved in complex atomic mech10 anisms such as the attachment-detachment of atoms or 11 the atomic diffusion at the periphery of nanostructures 12 [1 and 2]. To study the mass transfer mechanisms, differ13 ent experimental strategies have been carried out based 14 on the spatio-temporal fluctuations of the position of 15 isolated/interacting steps [3-5] or on the step displace16 ment velocity when a driving force intervenes using the 17 fluctuation-dissipation theorem [6-8]. In particular the 18 application of an electric current is known to bias the 19 diffusion of mobile adatoms. This effect, called electroso migration [9-15], can cause substantial changes in the 21 surface morphology such as step bunching for vicinal sur22 faces [16-19] or shape instabilities of 2D islands [20-24]. 23 However it has been recently recognized that an electric 24 current may not only impact adatom diffusion but also 25 atomic steps themselves by modifying their local proper26 ties such as the adatom equilibrium concentration close 27 to the step and/or the kinetic coefficients of attachment28 detachment at step edges [5, 25, and 26]. These effects 29 arise since the force acting on atoms depends on their sо local environment that differs at step edge, kink site or 31 on top of a terrace. These local modifications of step 32 properties are, to date, largely unknown whereas they 33 are suspected to be extremely strong [5 and 25]. More34 over a better understanding of the effects of the electric 35 current on surface mass transport gives also indirect in36 formation about the electric resistance of surfaces [27]. 37 Indeed the electric forces acting on atoms, kink sites and 38 step edges are compensated by opposite forces acting on 39 charge carriers caused by these surface structures. These 40 forces change the surface electric resistivity and may play 41 a major role in electrical conductors when down-scaling 42 in size [28 and 29]. This calls for specific studies on the 43 effect of an electric current on the step properties and 44 mass transport phenomena at the nanoscale.

45 In this letter we analyze quantitatively the atomic 46 mechanisms of mass transport and step properties on
${ }_{47} \mathrm{Si}(111)$ under an electric bias by addressing precisely the 48 boundary conditions to disentangle all the contributions. 49 In that purpose we have met two essential conditions: so (1) An advacancy island where atomic displacements oc51 cur at the interior of a confined $2 \mathrm{D}$ space closed by a 52 step edge; and (2) a driving force induced by an elec53 tric current to move the island. By adjusting the area 54 of the island and measuring its drift velocity induced 55 by an electric current we determine the mechanisms of 56 mass transfers. This study is based on an in operando 57 observation under an electric current of the $\mathrm{Si}(111)$ sur58 face with low energy electron microscopy (LEEM). The 59 experimental set-up allows to study the spatio-temporal 60 dynamics of mass transfers at atomic steps [30]. We show 61 a transition from a kinetics of mass transfer limited by 62 attachment-detachment of atoms at step edges for small 63 islands to a kinetics limited by terrace diffusion for large 64 islands. We deduce that the kinetic length for attach$65 \mathrm{ment} /$ detachment is $d \sim 500 \mathrm{~nm}$ and the effective va66 lence $\mathrm{Z}^{*}$ of the $\mathrm{Si}$ adatoms at the surface is $2.8 \pm 0.5$. Im67 portantly our detailed analysis of the stationary shape of 68 the electromigrating advacancy islands is consistent with 69 a strong modification of the local properties of attach$70 \mathrm{ment} /$ detachment at step edges induced by the electric 71 current.

72 The experiments were performed in an ultra high vac73 uum (UHV) setup equipped with a low energy electron 74 microscope (LEEM III, Elmitec GmbH) [30]. Si(111) 75 substrates ( $\mathrm{n}$ or $\mathrm{p}$-doped, $\rho=1 \Omega \mathrm{cm}$ ) were cut into pieces 76 of $15 \times 3 \times 0.5 \mathrm{~mm}^{3}$, cleaned with acetone and ethanol be77 fore introduction in UHV. An electric current is applied 78 through the sample via two Mo electrodes clamped to its 79 extremities. The samples were degassed in UHV for sevso eral hours at about $1100 \mathrm{~K}$ and then flashed above $1500 \mathrm{~K}$ 81 for a few seconds by direct current heating. Advacancy is82 lands are created by Si sublimation in the middle of large 8 s terraces [31]. The surface evolution under electromigra84 tion is studied by LEEM in bright field mode, with an 85 electron beam energy of $3 \mathrm{eV}$. To change the advacancy 86 islands size, Si was deposited in situ by a homemade ${ }_{87}$ direct current evaporator made of a piece of $\mathrm{Si}$ wafer s clamped between Mo electrodes. 


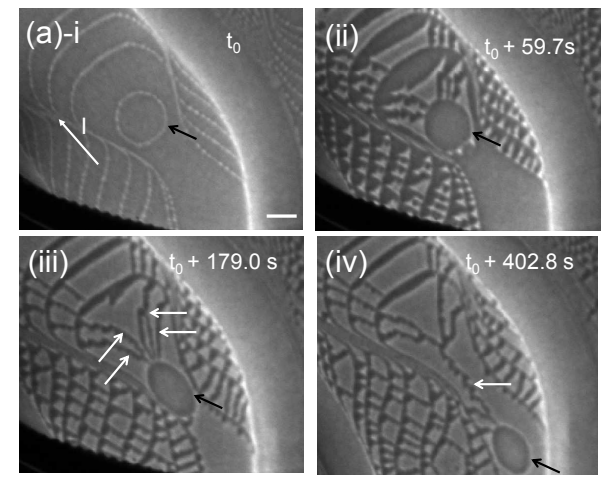

(b)

(c)

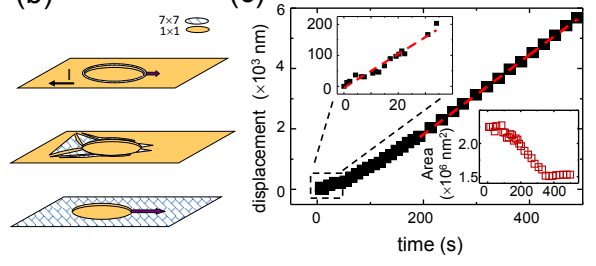

111 increases up to $13.1 \pm 0.1 \mathrm{~nm} \cdot \mathrm{s}^{-1}$ and reaches a station112 ary value when the $7 \times 7$ phase significantly covers the 113 surrounding surface. Simultaneously, after an initial size ${ }_{114}$ reduction due to mass transfers with the exterior, the is115 land size reaches also a steady state. Mass transfers have 116 two contributions: The Gibbs-Thompson effect favors the 117 capture of adatoms as the advacancy island curvature is 118 locally the largest one (in absolute). The phase transi119 tion expels the excess atoms of the $1 \times 1$ that diffuse to 120 the step edge [35]. The fact that the island area stabi121 lizes indicates that mass transfers from the exterior are 122 nearly entirely suppressed when the $7 \times 7$ covers most of 123 the surface. This diffusion barrier effect [36] is due to the 124 large surface diffusivity of $\mathrm{Si}$ adatoms on the $1 \times 1$ with 125 respect to the $7 \times 7$ (ratio $\sim 20,[7]$ ). During its displace126 ment the advacancy island can reach a step edge or a de127 fect that may induce the nucleation of the $7 \times 7$ inside the 128 island. To prevent this process from occurring the elec129 tric current direction is regularly reversed to change the 130 drift direction by electromigration while keeping a con131 stant temperature $( \pm 1 \mathrm{~K})$. The islands move back and 132 forth over a distance larger than $10 \mu \mathrm{m}$ on extended ter133 races without meeting any surface defect or step (Fig. $\left.{ }_{134} 2(\mathrm{~b})\right)$. Concomitantly this process allows for the disap${ }_{135}$ pearance of all $1 \times 1$ out-of-phase boundaries attached to 136 the islands by merging and detachment from the rear 137 side and by removal at the front side. Let us note that 138 a few out-of-phase boundaries have barely no effect on 139 the measured velocity but their removal is important to 140 determine the stationary shape of the advacancy island 141 without ambiguity.

FIG. 1. (a) Sequence of LEEM images during the $1 \times 1 \rightarrow$ $7 \times 7$ phase transition and under electric heating (see complete movie S1 in the supplementary materials). (i) Nucleation of $7 \times 7$ surface reconstruction at the step edges on the upper terraces. (ii) Spreading of the $7 \times 7$ onto the terraces except in the advacancy island (black arrow) where the $7 \times 7$ nucleation is hindered. (iii) Formation of $1 \times 1$ out-of-phase boundaries at the $7 \times 7$ domain intersections (white arrows). (iv) Migration of the advacancy island in the $\langle\overline{11} 2\rangle$ direction, opposite to the electric current. Out-of-phase boundaries merging at the rear side of the island. Electron energy $\mathrm{E}=3 \mathrm{eV}$. Scale bar $1 \mu \mathrm{m}$. (b) Scheme of the surface evolution under slow cooling. (c) Time evolution of the displacement of the advacancy island (black square). The steady velocity is $13.1 \pm 0.1 \mathrm{~nm} . \mathrm{s}^{-1}$ (velocity in (a)-i is $5.4 \pm 0.3 \mathrm{~nm} \cdot \mathrm{s}^{-1}$, see top inset) and the area is $1.5 \pm 0.110^{6} \mathrm{~nm}^{2}$ (see bottom inset).

9o LEEM images in Fig. 1(a) show the time evolution of 91 the $\mathrm{Si}(111)$ surface while crossing the $1 \times 1 \rightarrow 7 \times 7$ phase 92 transition temperature $(1133 \mathrm{~K})$. The low temperature ${ }_{93} 7 \times 7$ surface reconstruction nucleates at the step edges ${ }_{94}$ on the upper terraces [32] and appears as bright lines 95 (Fig. 1(a)-i). Upon slow cooling, by decreasing the elec96 tric current, the $7 \times 7$ phase extends onto the terraces ${ }_{97}$ (Fig. 1(a)-ii). Since the crystallographic arrangement 98 of the different $7 \times 7$ domains does not necessarily coin99 cide, $1 \times 1$ out-of-phase boundaries persist at their inter100 sections. Moreover the nucleation of the $7 \times 7$ phase is hin101 dered at the lower step edges and on terraces [7], therefore 102 the advacancy island in the middle of Fig. 1(a)-iii stays ${ }_{103}$ in a metastable supercooled $1 \times 1$ state [33]. This effect ${ }_{142}$ 104 was originally described as a hysteresis of the $1 \times 1 \longleftrightarrow{ }_{143}$ $1057 \times 7$ phase transition temperature [34]. Interestingly this ${ }_{144}$ 106 advacancy island migrates in the direction opposite to 145 107 the electric current (Fig. 1(a)-iii-iv). During the dis- 146 gration we have studied the size-dependence of the island 108 placement, the out-of-phase boundaries attached at the 147 velocity in the stationary regime. Figure 2(a) shows that 109 rear of the island merge from time to time and/or spon- ${ }_{148}$ the velocity increases with the island effective radius $R$ ${ }_{110}$ taneously detach. The velocity of the $a d$ vacancy island ${ }_{149}(R=\sqrt{A} / \pi$ where $A$ is the island area). Pierre-Louis et (a)

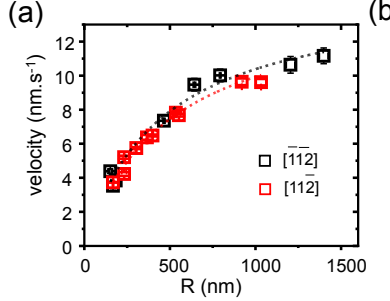

(c)

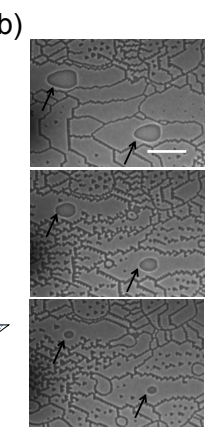

FIG. 2. (a) Advacancy island velocity versus radius. The islands electromigrate in the $\langle\overline{11} 2\rangle$ (black square) and $\langle 11 \overline{2}\rangle$ (red square) directions. Fit of the velocity (dotted lines). (b) LEEM images of islands of different sizes electromigrating in the $\langle 11 \overline{2}\rangle$ direction (scale bar $5 \mu \mathrm{m}$, see complete movie $\mathrm{S} 2$ in the supplementary materials). (c) Scheme of mass transfer process: detachment of atoms (D), biased terrace diffusion (TD) and attachment (A). To address the mass transport mechanisms that are occurring inside the advacancy islands under electromi- 
${ }_{150} \mathrm{al}$. [20] have analyzed the island velocity in the frame- 205 Einstein relation $v_{1 \times 1}=\frac{D_{1 \times 1}}{k_{B} T} F$ where $k_{B}$ is the Boltz151 work of the linear response theory with weak electromi- 206 mann constant, $T$ the temperature and $F=Z^{*} e E$ the 152 gration. Considering a kinetics of mass transport by at- 207 electromigration force. Therefore the force and the ef153 tachment (A), detachment (D) and terrace diffusion (TD) 208 fective charge $Z^{*}$ of Si adatoms can be obtained if the 154 inside the $1 \times 1$ advacancy island (see Fig. 2(c)), and ne- 209 diffusion coefficient $D_{1 \times 1}$ is known. Hibino et al. have 155 glecting the adatom flux from the upper terrace $(7 \times 7)$, 210 found $D_{1 \times 1} c_{e q}=3.010^{7} \mathrm{~s}^{-1}[7$ and 33] at the phase 156 the island drift velocity resulting from these processes is 211 transition temperature. Pang et al. have obtained by $157[20]$ :

$$
V_{i s l}=c_{e q} v_{1 \times 1} \frac{R}{R+d}
$$

158 where $c_{e q}$ is the equilibrium surface concentration of mo159 bile adatoms, $v_{1 \times 1}$ is the adatoms velocity on the $(1 \times 1)$ 160 terrace and $d=D_{1 \times 1} / k$ is the kinetic length of attach$161 \mathrm{ment} /$ detachment and is defined as the ratio of the sur162 face diffusion coefficient $D_{1 \times 1}$ to the rate $k$ of adatom 163 attachment to the step from the terrace. Let us note that 164 the mechanism of periphery diffusion of atoms along the 165 step edge has been neglected since the velocity should 166 decay as $1 / R[20]$ and no evidence of this behavior is 167 measured even for the smallest radius. The fit of the ex${ }_{168}$ perimental plots give two key parameters $c_{e q} v_{1 \times 1}$ and $d$. 212 different approaches $D_{1 \times 1} c_{e q}=2.0 \pm 0.210^{7} \mathrm{~s}^{-1}[8]$ in a 213 slightly higher temperature regime $(1163 \mathrm{~K})$. Considering $2 \mathbf{1 4}$ an average value for $D_{1 \times 1}$ we can deduce $F=1.4 \pm 0.3$ ${ }_{215} 10^{-6} \mathrm{eV} \cdot \mathrm{nm}^{-1}$ and the only free parameter, i.e. the ef216 fective charge of Si adatoms $Z^{*}=2.8 \pm 0.5(E=490$ ${ }_{217} \mathrm{~V} \cdot \mathrm{m}^{-1}$, atomic area: $\left.0.064 \mathrm{~nm}^{2}\right)$. The deduced value of ${ }_{218} Z^{*}$ is larger by one order of magnitude with earlier re${ }_{219}$ ports [38 and 39] except for [40] $\left(Z^{*}>1.3\right)$. The model 220 hypothesis of a weak electromigration is confirmed since 221 the available thermal energy is much larger than the en222 ergy to electromigrate $\frac{F a}{k_{B} T} \sim 10^{-5} \ll 1$ where $a=0.384$ $223 \mathrm{~nm}$ is the atomic lattice parameter [20]. The second term 224 that is deduced from the fit is the kinetic length of at225 tachment/detachment $d$. We obtain $d_{\langle 11 \overline{2}\rangle}=450 \pm 100$ $226 \mathrm{~nm}$ and $d_{\langle\overline{11} 2\rangle}=500 \pm 30 \mathrm{~nm}$ respectively for an island ${ }_{169}$ The first term is deduced from the asymptotic velocity at ${ }^{227}$ displacement in the $\langle 11 \overline{2}\rangle$ and the $\langle\overline{11} 2\rangle$ directions. It 170 large radius $\left(15 \pm 1 \mathrm{~nm} . \mathrm{s}^{-1}\right)$ and is only related to terrace ${ }^{228}$ is interesting to note that, contrary to the equilibrium 171 diffusion of the electromigrating adatoms. To estimate ${ }^{229}$ concentration that is close to the $1 \times 1$ surface, the kinetic 172 the $a d$ atom velocity $v_{1 \times 1}$ we have to determine first $c_{e q}$. ${ }^{230}$ length of attachment/detachment at the hybrid step edge ${ }_{173}$ Since the step edge is hybrid, $1 \times 1$ reconstructed on the ${ }^{231}$ is similar to the one measured at the step edges of the 174 lower terrace and $7 \times 7$ on the upper one, the equilib- ${ }^{232} 7 \times 7$ reconstructed surface [41]. To explain this kinetic 175 rium concentration of adatom close to the step edge is ${ }^{233}$ length we can note that, as for the $7 \times 7$ reconstructed 176 unknown. We have measured the adatom concentration ${ }^{234}$ surface, the advance of the hybrid step edge needs also 177 in the $1 \times 1$ advacancy island by decreasing the temper- ${ }^{235}$ to build $7 \times 7$ unit cells. This process is related to en178 ature to induce the $1 \times 1 \rightarrow 7 \times 7$ phase transition. The ${ }^{236}$ ergy barriers and probably to the occurrence of concerted 179 excess of adatoms expelled by the phase transition con- ${ }^{237}$ events that are necessary to achieve the complex mech180 densates at step edges and shrinks the $a d$ vacancy island ${ }^{2 \mathbf{3 8}}$ anisms involved in the formation of a $7 \times 7$ unit cell $[42$ 181 area (see supplementary materials S3). The area fraction ${ }^{239}$ and 43]. From the evaluation of $d$ we can estimate the 182 lost after the phase transition is $0.08 \pm 0.02$. Moreover $240 \mathrm{rate}$ of attachment/detachment at a step edge per atomic 183 considering that the $7 \times 7$ and the bulk-terminated $1 \times 1$ 241 site $k c_{e q} a=c_{e q} D_{1 \times 1} a / d \sim 1.9 \times 10^{4} \mathrm{~s}^{-1}$. It is also in184 structure have a difference of atomic density of 0.04 [35], 242 structive to estimate the average macroscopic time for ${ }_{185}$ we can estimate that the density of mobile adatoms of 243 adatoms to detach from the front side, cross the island ${ }_{186}$ the $1 \times 1$ is $0.12 \pm 0.02$. As the steps on $\mathrm{Si}(111)$ are bi- ${ }^{244}$ and attach at the rear side. The traveling time across ${ }_{187}$ layers this corresponds to $0.24 \pm 0.04$ monolayer (ML) 245 the terrace by diffusion is about $t_{d} \sim 2 R / v_{1 \times 1}$ and for a ${ }_{188}$ of adatoms on the $1 \times 1$ surface of the advacancy is- 246 typical island of $1 \mu \mathrm{m}$ radius $t_{d} \sim 15 \mathrm{~s}$. As a comparative ${ }_{189}$ land. This result is similar to 0.2 ML as estimated by 247 time scale, the delay time for an atom to make all attach190 [35] considering the $1 \times 1$ surface. This result is also ${ }^{248} \mathrm{ment} /$ detachment processes to cross the island is about 191 consistent with the fact that the equilibrium concentra- ${ }^{249} t_{A D} \sim 2 d / v_{1 \times 1} \sim 6$ to $8 \mathrm{~s}$. This indicates that many 192 tion of adatoms is a thermodynamic quantity. It is re- 250 events of (re)-attachment-detachment occur during this 193 lated to a difference of energy between two states: an ${ }^{252}$ traveling $\left(k c_{e q} a \times t_{A D} \sim 10^{5}\right.$, see Fig.2(c)).

194 atom attached at a step edge and on a terrace (adatom). ${ }_{253}$ In addition to velocity, the advacancy island shape in 195 As the chemical environments of an atom attached to a 254 the stationary regime is measured and depends both on ${ }_{196} 7 \times 7$ or $1 \times 1$ step edge are similar and very distinct 255 the island size and electric current direction. In Fig. 3(a), 197 from an adatom on top of a $1 \times 1$ terrace, we expect 256 the advacancy islands have a facetted front and an overall 198 that the step edge reconstruction only slightly modifies 257 triangular shape when they migrate in the $\langle 11 \overline{2}\rangle$ direction 199 the equilibrium concentration. Using our experimental 258 whereas they have a lozenge shape (elongated head and 200 result of $c_{e q}$ and correcting the velocity with $a d$ vection 259 lateral facets) in the opposite direction. If they move 201 [37] (sweeping effect on the adatoms due to the step mo- 260 in the $\langle\overline{1} 10\rangle$ direction the shape is no more symmetric 202 tion) we get finally the adatom velocity $v_{1 \times 1}=110 \pm 8{ }_{261}$ (Fig. 3(d)). In all cases the shape is elongated in the ${ }_{203} \mathrm{~nm} . \mathrm{s}^{-1}$ on the $1 \times 1$ surface reconstruction at the phase 262 migration direction. This elongation increases with the 204 transition temperature. This velocity derives from the 263 island size and tends to be circular for small sizes (the 
(a)

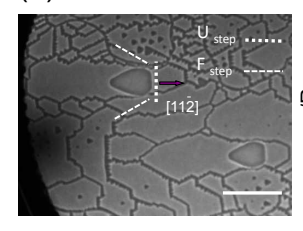

(b)
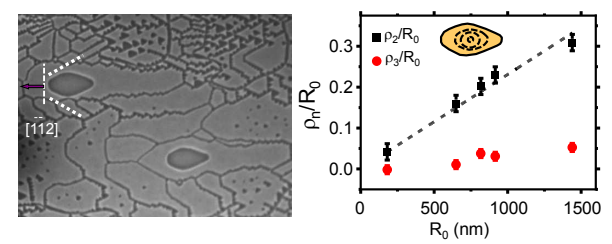

(c)

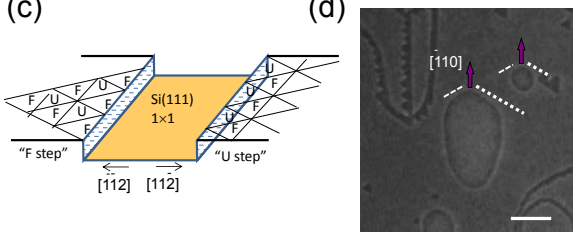

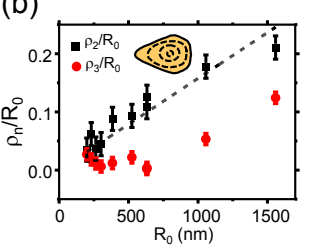

(d)

FIG. 3. (a) LEEM images of advacancy islands electromigrating in the $\langle 11 \overline{2}\rangle$ (top) and the $\langle\overline{11} 2\rangle$ (bottom) directions (scale bar $5 \mu \mathrm{m}$ ). The shape is respectively a triangle and a lozenge. Unfaulted steps $\left(\mathrm{U}_{\text {step }}\right)$ are shown as dotted lines and faulted steps $\left(\mathrm{F}_{\text {step }}\right)$ as dashed lines [44]. (b) Fourier coefficients of the island shape as function of island radius for both direction. (c) Scheme of the $U$ and $F$ step edge structure. (d) LEEM image of two advacancy islands electromigrating in the $\langle 1 \overline{1} 0\rangle$ direction. The shape is asymmetric (scale bar 1 $\mu \mathrm{m}$, see complete movie $\mathrm{S} 4$ in the supplementary materials).

264 typical crossover is about the attachment-detachment ki265 netic length $d$ ). To describe the island shape, we use the 266 polar coordinates $R(\theta)=R_{0}+\rho(\theta)$ ( $R_{0}$ is the mean ra267 dius), and we apply the Fourier series expansion of $\rho(\theta)$ :

$$
\rho(\theta)=\sum_{n \geq 2}^{\infty} \rho_{n} \cos (n \theta)+\nu_{n} \sin (n \theta)
$$

268 where $\rho_{n}$ and $\nu_{n}$ are the Fourier coefficients $\left(\nu_{n}=0\right.$ for 269 symmetric islands and $\rho_{1}$ and $\nu_{1}$ are not considered be270 cause they correspond to a simple shape translation). In ${ }_{271}$ Fig. 3(b) are plotted the normalized Fourier coefficients $272 \rho_{n} / R_{0}$ as function of the island radius $R_{0}$ when the is273 lands are migrating along the $\langle 11 \overline{2}\rangle$ and $\langle\overline{11} 2\rangle$ directions. 274 The main term of elongation is the $\mathrm{n}=2$ mode $\rho_{2} / R_{0}$ 275 and in both cases it increases approximately linearly with 276 the island radius. $\rho_{2} / R_{0}$ is larger when the island has a 277 lozenge shape. The triangular shape of the islands mi278 grating in the $\langle 11 \overline{2}\rangle$ direction is given by a strong $\mathrm{n}=3$ 279 mode $\rho_{3} / R_{0}$ that is increasing non-linearly with the is280 land radius.

281 Our first insight into the island shape and symmetry $\mathbf{2 8 2}$ is based on crystallographic considerations. The step $\mathbf{2 8 3}$ edge properties of the $7 \times 7$ have a threefold symmetry 284 [45 and 46]. However due to symmetry breaking by elec$\mathbf{2 8 5}$ tromigration, maximum a mirror symmetry can be ex-

$2 \mathbf{2 6}$ pected. As observed experimentally if the electric cur${ }_{287}$ rent is along the symmetry axis $\langle 11 \overline{2}\rangle$ the shape has a 288 mirror line whereas it is not the case when the electric 289 current is along the non-symmetric $\langle\overline{1} 10\rangle$ direction (Fig. $2903(\mathrm{~d}))$. As the shape is far from equilibrium the kinetic 291 of mass transfers such as the one involved in the attach292 ment/detachment of atoms at step edges is expected to 293 play a major role.

294 In the framework of a continuous step model with 295 isotropic surface properties, the shape of advacancy is296 lands driven by an electromigration force on adatoms and 297 considering mass transfers by terrace diffusion and at298 tachment/detachment at step edges has been calculated 299 [20, 24, and 37]. The elongation of the advacancy islands 300 is perpendicular to the migration direction. This shape 301 can be qualitatively interpreted as resulting from a mass 302 flux towards the migration axis. Indeed in presence of 303 a slow kinetics of attachment, the adatoms make several 304 trials before attaching to the step and have a residual 305 drift towards the migration axis. In a steady state the 306 local curvature of the island is modified to compensate 307 this mass flux by a capillary effect. Quantitatively the 308 change of shape involves the $n=2$ mode as $\rho_{2} / R_{0}$ ratio зоя (elongation) and reads $\left([20]\right.$ for $\left.d \ll R_{0}\right)$ :

$$
\frac{\rho_{2}}{R_{0}}=-\frac{1}{12 \Gamma} \frac{R_{0}^{2}}{\xi^{2}} d<0
$$

310 where $\Gamma=\frac{a^{2} \tilde{\beta}}{k_{B} T}$ is the capillary length (Gibbs-Thomson $\mathbf{3 1}$ effect), $\tilde{\beta}$ is the step edge stiffness and $\xi$ is a charac$\mathbf{3 1 2}$ teristic length associated with the electromigration force ${ }_{313}\left(\xi=\frac{k_{B} T}{F}=70 \mu \mathrm{m}\right)$. This result is opposite to the ex314 perimental shape since the elongation of the advacancy 315 islands is along the migration direction $\left(\frac{\rho_{2}}{R_{0}}>0\right)$. As this 316 result is observed whatever the direction of the electric 317 current we infer that even if the modeling could include $\mathbf{3 1 8}$ the anisotropy of the surface properties, it alone could 319 not explain that the shape is always elongated along the 320 migration axis. Therefore we propose that the electric 321 current modifies not only the adatom displacement but 322 also the atomic step properties. As a minimum model, 323 the electric current breaks the threefold symmetry of the 324 kinetic rate of attachment-detachment at the step edges 325 [47]. To study this effect we expand the kinetic length $d$ 326 as a Fourier series $d=\bar{d}+\sum_{n} d_{n} \cos (n \theta)$ where $\bar{d}$ is the 327 mean kinetic length of attachment/detachment and $d_{n}$ 328 are the Fourier coefficients for a symmetric shape. The 329 main Fourier term acting as an electrobias, i.e. chang3 зо ing the kinetics of attachment-detachment at the step 331 edge, is expected to be $d_{1}$ since it breaks the symmetry 332 between the island front where the current is step-down 333 and the island rear where the current is step-up. Let 334 us note that without electrobias only $d_{3 n}$ exists by sym335 metry. Expanding linearly the shape of the advacancy 336 island with this electrobias effect we obtain:

$$
\frac{\rho_{2}}{R_{0}}=-\frac{1}{12 \Gamma}\left[\frac{R_{0}^{2}}{\xi^{2}}\left(\bar{d}+\frac{d_{2}}{2}\right)-2 \frac{R_{0}}{\xi}\left(d_{1}+d_{3}\right)\right]
$$


337 The shape elongation $\frac{\rho_{2}}{R_{0}}$ shows a new contribution that 36 similar electrobias effect has also been found. However 338 increases linearly with the island radius $R_{0}$ as in the mea- 368 the studies on Ag metal [5 and 25] have addressed a dif339 surements, and is along the migration axis if $d_{1}+d_{3}>0$. 369 ferent regime of mass transport dominated by atomic dif340 As $\frac{R_{0}}{\xi} \sim 0.014 \ll 1$ we can neglect the second order con- 370 fusion along the island periphery. The electrobias effect 341 tribution in eq. (4). To estimate only the electrobias $\mathbf{3 7 1}$ was studied considering a different methodology based on 342 effect $d_{1}$ we use the change of the current direction in $\mathbf{3 7 2}$ the analysis of step fluctuations and island velocity but 343 the experiment. Assuming that $d_{3}$ is not significantly af- $\mathbf{3 7 3}$ not on the island shape whereas it is strongly sensitive to 344 fected by the electrobias effect, since it is a three order $\mathbf{3 7 4}$ the local modifications of the step edge properties [20].

345 term in the series expansion and do not coincide with 346 the symmetry of the electric current, the inversion of ${ }_{347}$ the current direction changes $d_{3}$ by $-d_{3}$ [48]. There348 fore the kinetic length of electrobias $d_{1}$ is obtained by 349 averaging both shape elongation $\frac{\rho_{2}}{R_{0}}$ in the $\langle 11 \overline{2}\rangle$ and 37 In conclusion we have shown on $\mathrm{Si}(111)$ surface that 376 an advacancy island in the $1 \times 1$ high temperature phase 377 and surrounded by the $7 \times 7$ low temperature phase can be 378 stabilized. This regime allows keeping the $2 \mathrm{D}$ island in a 379 confined state in terms of atomic exchanges. Then under s50 the $\langle\overline{11} 2\rangle$ directions. We estimate that $d_{1} \sim 83 \pm 12$ з8о the influence of an electric current, the island is moving. $3 \mathbf{n 1} \mathrm{nm}(\Gamma=1 \mathrm{~nm}[8])$ and considering that this contribution $\mathbf{3 8 1}$ The analysis of the velocity and shape of the island as $3 \mathbf{2 2}$ is thermally activated, we extract the activation energy $\mathbf{3 8 2}$ function of its radius show that (i) Si adatoms migration ${ }_{353} E_{1}=k_{B} T \ln \left(1+\frac{d_{1}}{\bar{d}}\right)=1.510^{-2} \mathrm{eV}$. This electrobias ef- ${ }^{383}$ on the terrace is biased and they have an effective valence 354 fect on the step edge is much larger than on adatoms $355\left(E=F a / 2 \sim 2.610^{-7} \mathrm{eV}\right)$. This result could be related $\mathbf{3 5 6}$ to an intrinsic change of step properties induced by the 357 current but it may also arise from a change of kink den35 sity at step edges. Indeed it has been shown [49] that an 359 electric current in the $\langle 11 \overline{2}\rangle$ direction along a step and as360 cending the kinks favors the formation of an atomically 361 straight step edge. Therefore considering that the kinet362 ics of mass transfers at step edges is mediated by kinks 363 then the rate of attachment/detachment could be indeed 364 strongly modified by the electric current. Such an electro- 394 discus bias effect on step edges or kink 作 fochnical support. This work has been supported by 366 to occur on semiconductor surfaces [50]. On metals a 396 the ANR grant HOLOLEEM (ANR-15-CE09-0012). $42{ }^{11}$ A. H. Verbruggen. IBM J. Res. Dev., 32(1):93-98, 1988.

* leroy@cinam.univ-mrs.fr

Hyeong-Chai Jeong and Ellen D Williams. Surf. Sci. Rep., 424 34(6-8):171-294, 1999.

2 Chaouqi Misbah, Olivier Pierre-Louis, and Yukio Saito. 4 Reviews of Modern Physics, 82(1):981-1040, 2010.

N. C. Bartelt, J. L. Goldberg, T. L. Einstein, Ellen D. 4 Williams, J. C. Heyraud, and J. J. Métois. Physical Review 420 $B, 48(20): 15453-15456,1993$.

${ }^{4}$ DB Dougherty, I Lyubinetsky, ED Williams, M Con- 4 stantin, C Dasgupta, and S Das Sarma. Physical Review Letters, 89(13):136102, 2002.

${ }^{5}$ O. Bondarchuk, W. G. Cullen, M. Degawa, E. D. Williams, T. Bole, and P. J. Rous. Physical Review Letters, 99(20):206801, 2007.

6 K. Thurmer, J.E. Reutt-Robey, E.D. Williams, M. Uwaha, 4 A. Emundts, and H.P. Bonzel. Physical Review Letters, 87(18):186102, 2001.

H. Hibino, C.-W. Hu, T. Ogino, and I. S. T. Tsong. Physical Review B, 63(24):245402, 2001.

vich, F. Szalma, and T. L. Einstein. Physical Review B 77(11):115424, 2008.

H. B. Huntington and A. R. Grone. J. Phys. Chem. Solids, 20:76, 1961.
$384 Z^{*}$ of $2.8 \pm 0.5$ (ii) the kinetic of attachment/detachment 385 of atoms at the step edges is very slow and we evaluate 386 the kinetic length as $\sim 500 \mathrm{~nm}$. (iii) An electrobias effect 387 on the kinetics of attachment/detachment at step edges 388 elongates the island shape in the direction of the electric 389 current. We believe that a complete modeling including 390 all the effects of anisotropy, non-linearities and high den391 sity of adatoms would be necessary to describe precisely 392 the island shape.

We are grateful to Olivier Pierre-Louis for instructive
${ }_{423}{ }^{12}$ P. S. Ho and T. Kwok. Rep. Prog. Phys., 52(3):301-348, 1989.

${ }_{425}^{13}$ H. Yasunaga and A. Natori. Surf. Sci. Rep., 15(6-7):205280, 1992.

${ }_{427}^{14}$ S. Curiotto, F. Cheynis, P. Müller and F. Leroy. ACS Appl. Nano Mater., 3(2):1118-1122, 2020.

${ }^{15}$ S. Curiotto, P. Müller, A. El Barraj, F. Cheynis, O. PierreLouis and F. Leroy. Appl. Surf. Sci., 469:463-470, 2019.

4316 A.V. Latyshev, A.L. Aseev, A.B. Krasilnikov, and S.I. Stenin. Surface Science, 213(1):157-169, 1989.

${ }_{433}{ }^{17}$ Y Homma, RJ McClelland, and H Hibino. Jpn. J. Appl. Phys., 29(12):L2254-L2256, 1990.

${ }_{435}^{18}$ F. Leroy, P. Müller, J. J. Metois, and O. Pierre-Louis. Phus. Rev. B, 76(4), 2007.

${ }_{437}^{19}$ F. Leroy, D. Karashanova, M. Dufay, J. M. Debierre, T. Frisch, J. J. Metois, and P. Müller. Surf. Sci., 603(3):507-512, 2009.

${ }_{440} 20$ O. Pierre-Louis and T. L. Einstein. Phys. Rev. B, $441 \quad 62(20): 13697-13706,2000$.

${ }_{442}{ }^{21}$ Philipp Kuhn, Joachim Krug, Frank Hausser, and Axel Voigt. Physical Review Letters, 94(16):166105, 2005.

${ }_{444}^{22}$ A. Kumar, D. Dasgupta, C. Dimitrakopoulos, and D. Maroudas. Appl. Phys. Lett., 108(19):193109, 2016.

${ }_{446}{ }^{23}$ A. Kumar, D. Dasgupta, and D. Maroudas. Physical Re447 view Applied, 8(1):014035, 2017.

${ }_{448}{ }^{24}$ Stefano Curiotto, Frédéric Leroy, Pierre Müller, Fabien 
449 Cheynis, Ali El-Barraj, Michail Michailov, and Bogdan 480

450 Ranguelov. J. Cryst. Growth, 520:42-45, 2019.

${ }_{451} 25$ C. Tao, W. G. Cullen, and E. D. Williams. Science, ${ }_{482}{ }^{38}$ $452328(5979): 736-740,2010$.

${ }_{453}{ }^{26}$ Kirk H. Bevan, Hong Guo, Ellen D. Williams, and Zhenyu ${ }_{484} 39$

454 Zhang. Physical Review B, 81(23):235416, 2010.

${ }_{455} 27$ E. D. Williams, O. Bondarchuk, C. G. Tao, W. Yan, W. G.

456 Cullen, P. J. Rous, T. Bole. New Journal of Physics, ${ }_{487}$

$4579(10): 387,2007$.

${ }_{458}{ }^{28}$ Bruno V.â C. Martins, Manuel Smeu, Lucian Livadaru, 489

459 Hong Guo, and Robert A. Wolkow. Physical Review Let- 490 460 ters, 112(24):246802, 2014.

${ }_{461} 29$ Sven Just, Marcus Blab, Stefan Korte, Vasily Cherepanov,

462 Helmut Soltner, and Bert VoigtlÄnder. Physical Review ${ }^{493}$

463 Letters, 115(6):066801, 2015

${ }_{464}^{30}$ F. Cheynis, F. Leroy, A. Ranguis, B. Detailleur, P. Bindzi, 495

465 C. Veit, W. Bon, and P. Müller. Review of Scientific In- 49 466 struments, 85:043705, 2014.

${ }_{467}{ }^{31}$ Yoshikazu Homma, Hiroki Hibino, Toshio Ogino, and ${ }_{498}$

468 Noriyuki Aizawa. Physical Review B, 55(16):R10237- 499

469 R10240, 1997.

${ }_{470}{ }^{32} \mathrm{~N}$ Osakabe, Y Tanishiro, K Yagi, and G Honjo. Surface 501

471 Science, 109:359-366, 1981.

47233 H. Hibino, Y. Watanabe, C.-W. Hu, and I. S. T. Tsong. 503

$473 \quad$ Physical Review B, 72(24):245424, 2005.

${ }_{474} 34$ C.-W. Hu, H. Hibino, T. Ogino, and I. S. T. Tsong. Surface 505

475 Science, 487:191-200, 2001.

${ }_{476}{ }^{35}$ Y.-N. Yang and E. D. Williams. Physical Review Letters, 507

$477 \quad 72(12): 1862-1865,1994$.

${ }_{478}^{36}$ H. Hibino, C.-W. Hu, T. Ogino, and I. S. T. Tsong. Phys- 509

479 ical Review B, 64(24):245401, 2001.
37 Frank Haußer, Philipp Kuhn, Joachim Krug, and Axel Voigt. Physical Review E, 75(4):046210, 2007.

Daniel Kandel and Efthimios Kaxiras. Physical Review Letters, 76(7):1114-1117, 1996.

9 E.S. Fu, D.J. Liu, M.D. Johnson, J.D. Weeks, and E.D. Williams. Surface Science, 385:259-269, 1997.

40 Andrés Saùl, Jean-Jacques Métois, and Alain Ranguis. Physical Review B, 65(7):075409, 2002.

41 W. F. Chung and M. S. Altman. Physical Review B, 66(7):075338, 2002.

${ }^{42}$ Wataru Shimada, Tomoshige Sato, and Hiroshi Tochihara. Physical Review B, 94(3):035402, 2016.

43 Ing-Shouh Hwang, Mon-Shu Ho, and Tien T Tsong. Physical Review Letters, 83(1):120, 1999.

44 H. Tochihara, W. Shimada, M. Itoh, H. Tanaka, M. Udagawa, and I. Sumita. Physical Review B, 45(19):11332$11335,1992$.

${ }^{45}$ Noriko Akutsu and Yasuhiro Akutsu. Journal of Physics: Condensed Matter, 11(35):6635-6652, 1999.

46 Noriko Akutsu. Surface Science, 630:109-115, December 2014.

${ }_{501}^{47}$ Nobuo Suga, Junya Kimpara, Nan-Jian Wu, Hitoshi Yasunaga, and Akiko Natori. Japanese Journal of Applied Physics, 39(Part 1, No. 7B):4412-4416, July 2000.

${ }_{04}^{48}$ Considering the general case where $d_{3}$ is also affected by the electric current we obtain by current reversal the quantity $d_{1}+d_{3}^{e l}$ in Eq.(4) where $d_{3}^{\text {el }}$ is the specific contribution of the electric current on $d_{3}$.

${ }_{508}{ }^{49}$ S. Yoshida, T. Sekiguchi, and K. M. Itoh. Applied Physics Letters, 87(3):031903, 2005.

${ }_{510} 50$ Mon-Shu Ho, Ing-Shouh Hwang, and Tien T. Tsong. Phys511 ical Review Letters, 84(25):5792-5795, 2000. 